\title{
Comparative anomalous small-angle x-ray scattering study of hotwire and plasma grown amorphous silicon-germanium alloys
}

\author{
G. Goerigk ${ }^{\text {a) }}$ \\ Institut für Festkörperforschung, Forschungszentrum Jülich, Postfach 1913, D-52425 Jülich, \\ Federal Republic of Germany \\ D. L. Williamson \\ Department of Physics, Colorado School of Mines, Golden, Colorado 80401
}

(Received 28 June 2001; accepted for publication 28 August 2001)

\begin{abstract}
The nanostructure of hydrogenated amorphous silicon-germanium alloys, $a-\mathrm{Si}_{1-x} \mathrm{Ge}_{x}: \mathrm{H}$, prepared by the hotwire deposition technique $(x=0.06-0.79)$ and by the plasma enhanced chemical vapor deposition technique $(x=0$ and 0.50$)$ was analyzed by anomalous small-angle x-ray scattering experiments. For all alloys with $x>0$ the Ge component was found to be inhomogeneously distributed with correlation lengths of about $1 \mathrm{~nm}$. A systematic increase of the separated scattering was found due to the increasing Ge concentration. The different preparation techniques show significant differences in the Ge distribution. (C) 2001 American Institute of Physics.
\end{abstract}

[DOI: $10.1063 / 1.1413488$ ]

\section{INTRODUCTION}

Hydrogenated amorphous silicon-germanium alloys, $a-\mathrm{Si}_{1-x} \mathrm{Ge}_{x}: \mathrm{H}$, play an important role in amorphous siliconbased solar cell technology. The Ge is added to produce lower band-gap material in multilayer devices to absorb the longer wavelength photons of the solar spectrum, leading to the highest conversion efficiency yet reported for this thinfilm technology. ${ }^{1}$ Values of $x$ near 0.5 are needed to lower the band gap to appropriate levels. However, Ge alloying systematically degrades the optoelectronic properties with increasing $x$ relative to the base $a-\mathrm{Si}: \mathrm{H}$ material. ${ }^{2,3}$ This degradation is likely due to a complex mixture of intrinsic (atomic scale potential fluctuations, intrinsic defects, preferential $\mathrm{H}$ bonding) and extrinsic (nonoptimized deposition conditions, poor nanostructure, Ge nonuniformity) problems based on a wide array of optoelectronic and structural measurements. It is typical that the nanostructure of $a-\mathrm{Si}_{1-x} \mathrm{Ge}_{x}: \mathrm{H}$ prepared by the most common method of plasma-enhanced chemical-vapor deposition (PECVD) is systematically more heterogeneous with increasing $x$ based on extensive small-angle $\mathrm{x}$-ray scattering (SAXS) studies. $^{4-7}$ The primary origin of this heterogeneity is the formation during growth of microvoids and columnar-like structures with low density regions around the columns. However, recent application of the synchrotron-based technique of anomalous small-angle x-ray scattering (ASAXS) has allowed the separation of the scattering due specifically to $\mathrm{Ge}$ composition fluctuations from those associated with microvoids or surface roughness. ${ }^{8,9}$ For most of the PECVD films examined, there was a significant Ge inhomogeneity and detailed analysis has led to a two-phase model with information on the size scale of the Ge composition fluctuations and the

\footnotetext{
a) Author to whom correspondence should be addressed; present address: DESY-HASYLAB, Notkestr. 85, D-22603 Hamburg, Federal Republic of Germany; electronic mail: guenter.goerigk@desy.de
}

difference in Ge content of the two phases. ${ }^{9}$ It was found that the use of hydrogen dilution during deposition, typically used to improve the optoelectronic properties and device performance, ${ }^{1,6}$ also led to improved homogeneity of the Ge. Another important result was that one film grown under a high ion bombardment condition showed no evidence of $\mathrm{Ge}$ nonuniformity. ${ }^{8}$ Similar results were obtained by SAXS from high $x$ alloys subjected to more ion bombardment during growth. ${ }^{7}$ Thus both the Ge-related heterogeneity and the void-related nanostructure can be improved by suitable deposition conditions.

There has been a growing interest in an alternate film deposition method for the $a$-Si:H-based materials, the hotwire chemical-vapor deposition (HWCVD) technique, ${ }^{10,11}$ due to evidence of improved stability ${ }^{12}$ and its potential for higher deposition rates while maintaining optoelectronic properties comparable to those of PECVD materials. ${ }^{13}$ The latter feature is particularly important from a manufacturing standpoint. Here we report on ASAXS results from a series of $a-\mathrm{Si}_{1-x} \mathrm{Ge}_{x}: \mathrm{H}$ alloy films made by HWCVD. Some optoelectronic properties and SAXS results have shown behavior similar to PECVD alloys. ${ }^{14,15}$ For comparison, two PECVD films prepared under conditions that were used for the record solar cell ${ }^{1}$ are also examined.

\section{EXPERIMENT}

The series of HWCVD films $(x=0.06-0.79)$ was made at NREL (National Renewable Energy Laboratory) using deposition conditions described elsewhere along with some optoelectronic properties. ${ }^{14}$ The two PECVD films were made by USSC (United Solar Systems Corporation) using deposition conditions nominally identical to those employed to produce the top $(x=0)$ and bottom $(x=0.50)$ intrinsic layers of a record efficiency triple-junction solar cell. ${ }^{1}$ All films were deposited on high-purity, 10- $\mu$ m-thick Al foils, which were then folded into four layers (HWCVD) or eight 
TABLE I. Anomalous dispersion corrections of Si and Ge (Refs. 17 and 18).

\begin{tabular}{lccccc}
\hline \hline No. & $E[\mathrm{keV}]$ & $f_{\mathrm{Ge}}^{\prime}$ & $f_{\mathrm{Ge}}^{\prime \prime}$ & $f_{\mathrm{Si}}^{\prime}$ & $f_{\mathrm{Si}}^{\prime \prime}$ \\
\hline 1 & 10.0084 & -2.057 & 0.596 & 0.181 & 0.216 \\
2 & 11.0950 & -6.963 & 0.494 & 0.155 & 0.176 \\
\hline \hline
\end{tabular}

layers (PECVD) for the ASAXS measurements. The thicker HWCVD films $(\sim 2 \mu \mathrm{m})$ compared to the PECVD films $(\sim 1$ $\mu \mathrm{m})$ allowed fewer layers. The deposition rate for the HWCVD material $(\sim 0.5 \mathrm{~nm} / \mathrm{s})$ was about five times that of the PECVD films. The Ge concentrations of the films under investigation were determined by electron probe microanalysis with an accuracy in $x$ of \pm 0.02 .

The ASAXS measurements were carried out at the JUSIFA beamline at HASYLAB, DESY Hamburg. ${ }^{16}$ Measurements were made for each sample with a twodimensional detector at two energies. A $q$ range from about 0.1 to $7 \mathrm{~nm}^{-1}$ was covered, where $q=(4 \pi / \lambda) \sin \theta(\lambda=\mathrm{x}$-ray wavelength and $2 \theta=$ scattering angle). Table I provides the anomalous dispersion corrections for $\mathrm{Si}$ and $\mathrm{Ge}$ at the two energies used here based on the calculations of Cromer and Liberman. ${ }^{17,18}$ These were used to permit the separation of the Ge-related scattering described in detail by Refs. 8 and 9 . Constant background effects due to diffuse scattering ${ }^{5}$ and due to Ge-fluorescence occurring near the $K$-absorption edge at $11.103 \mathrm{keV}$ have been subtracted from the measured intensities. The scattering intensity is calibrated into macroscopic scattering cross sections in units of cross-section per unit volume $\left[\mathrm{cm}^{2} / \mathrm{cm}^{3}=\mathrm{cm}^{-1}\right]$.

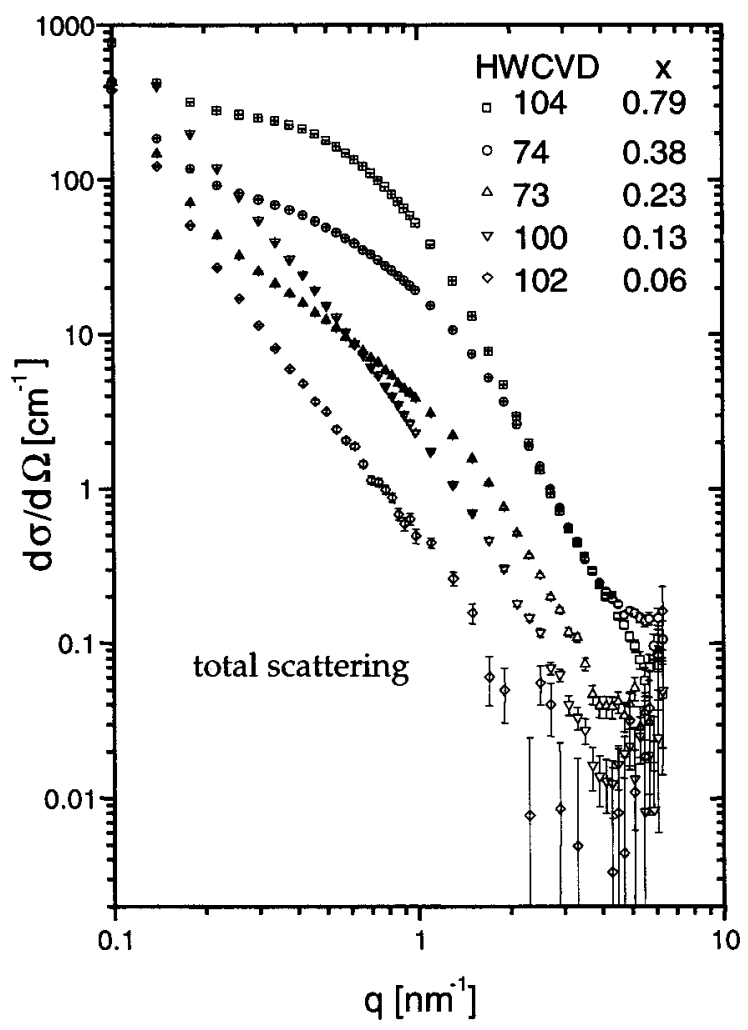

FIG. 1. Total scattering intensities at $10.010 \mathrm{keV}$ from the HWCVD films with different Ge content $(x)$.

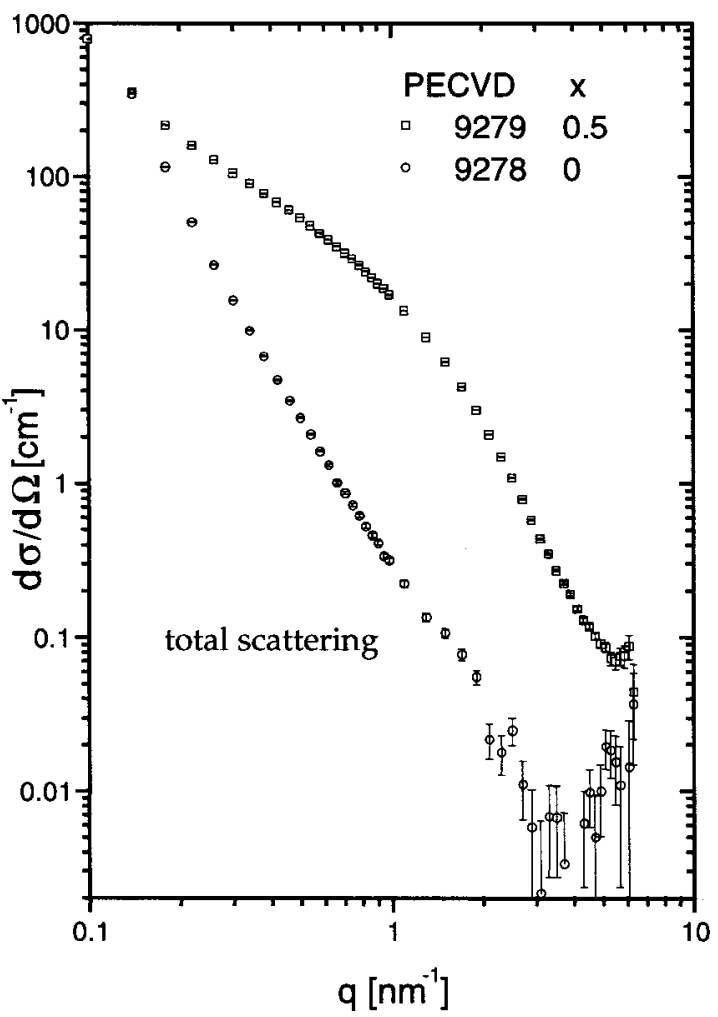

FIG. 2. Total scattering intensities at $10.010 \mathrm{keV}$ from PECVD films with no Ge and with $x=0.50$.

\section{RESULTS}

A brief review of the relevant expressions of anomalous small-angle x-ray scattering in connection with an early correlation model proposed by Debye et al. ${ }^{19}$ is presented in Refs. 8 and 9. Figures 1-4 show the scattering curves of the HWCVD alloys with $x=0.06,0.13,0.23,0.38$, and 0.79 and the two PECVD materials with $x=0$ and 0.5. In Figs. 1 and 2 the scattering curves represent the total scattering obtained from the alloys at the energy of $10010 \mathrm{eV}$. The scattering curves of Figs. 3 and 4 represent the separated scattering obtained from measurements at the two energies 10010 and $11091 \mathrm{eV}$.

The alloys clearly reveal a significant contribution from nonuniformly distributed $\mathrm{Ge}$ in the amorphous matrix. The solid lines passing through the separated scattering curves are fitted model functions according to the $q$ dependence described by Eq. (1). Equation (1) represents the correlation model of a two phase mixture with an additional $q^{-4}$ term describing some type of large inhomogeneity: ${ }^{20}$

$$
\Delta \frac{d \sigma^{\mathrm{tot}}}{d \Omega}\left(q, E_{1,2}\right)=\frac{b a^{3}}{\left(1+a^{2} q^{2}\right)^{2}}+\frac{c}{q^{4}} .
$$

For $q$ values $<0.3 \mathrm{~nm}^{-1}$ the separated scattering curves show some influence of scattering from some type of larger structures $(>20 \mathrm{~nm})$. The results for all samples obtained from the fits are summarized in Table II. The values of $a$, between 0.8 and $1.1 \mathrm{~nm}$, represent the correlation lengths ${ }^{19}$ obtained from the fits of the separated scattering curves, while $b$ gives the values of the extrapolated scattering at $q=0$ and represents the degree of inhomogeneity due to Ge. Figure 4(b) 


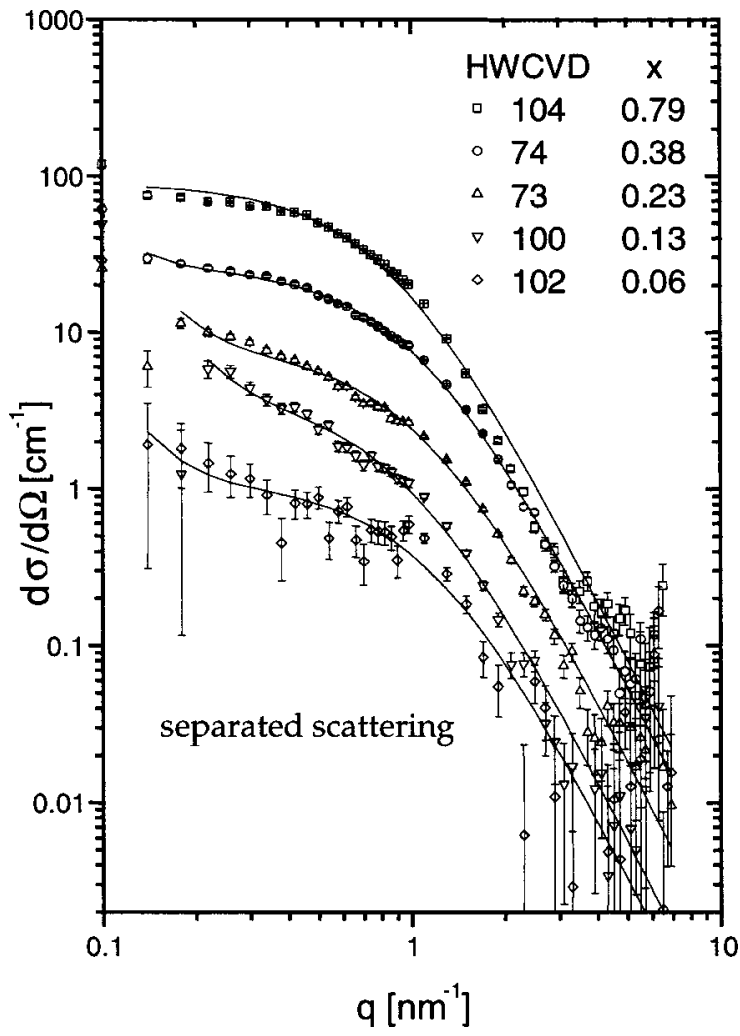

FIG. 3. Separated scattering intensities from HWCVD films with different Ge contents $(x)$.

(note the conversion of vertical axis to a linear scale) represents the separated scattering of PECVD $a-\mathrm{Si}: \mathrm{H}$, which scatters around zero intensity as expected due to $x=0$.

\section{DISCUSSION}

For all hotwire-deposited alloys significant contributions from nonuniform $\mathrm{Ge}$ in the amorphous matrix was found, even for the lower concentrations $(x=0.06,0.13$, and 0.23$)$. In comparison, former results obtained from PECVD material with $x=0.15$ showed no significant separated scattering of Ge inhomogeneities. ${ }^{9}$ The amount of the separated scattering increases monotonically with the $x$ due to an increasing amount of $\mathrm{Ge}$ inhomogeneities. The total scattering increases over half an order of magnitude when changing $x$ from 0.06 to 0.13 , while the shape of the scattering curve changes only slightly and is following roughly a $q^{-3}$ behavior. Upon increasing $x$ to 0.23 a dramatic change takes place. The total scattering intensity at $q<1 \mathrm{~nm}^{-1}$ is strongly reduced, while the total scattering intensity at the higher $q$ values continues to increase. The shape of the scattering curve now shows a more complicated behavior and is different from a simple power law. This tendency continues for $x=0.38$ with the scattering curve showing higher values of the scattering cross section due to the higher amount of inhomogeneities. Finally at $x=0.79$ again the shape of the total scattering curve changes. The scattering curve at $q$ values $q$ $<2 \mathrm{~nm}^{-1}$ is shifted to higher values of the cross sections indicating a higher amount of inhomogeneity, but at higher $q$ values the scattering curves of the samples with $x=0.38$ and 0.79 nearly coincide and show a $q^{-4}$ dependence, indicating

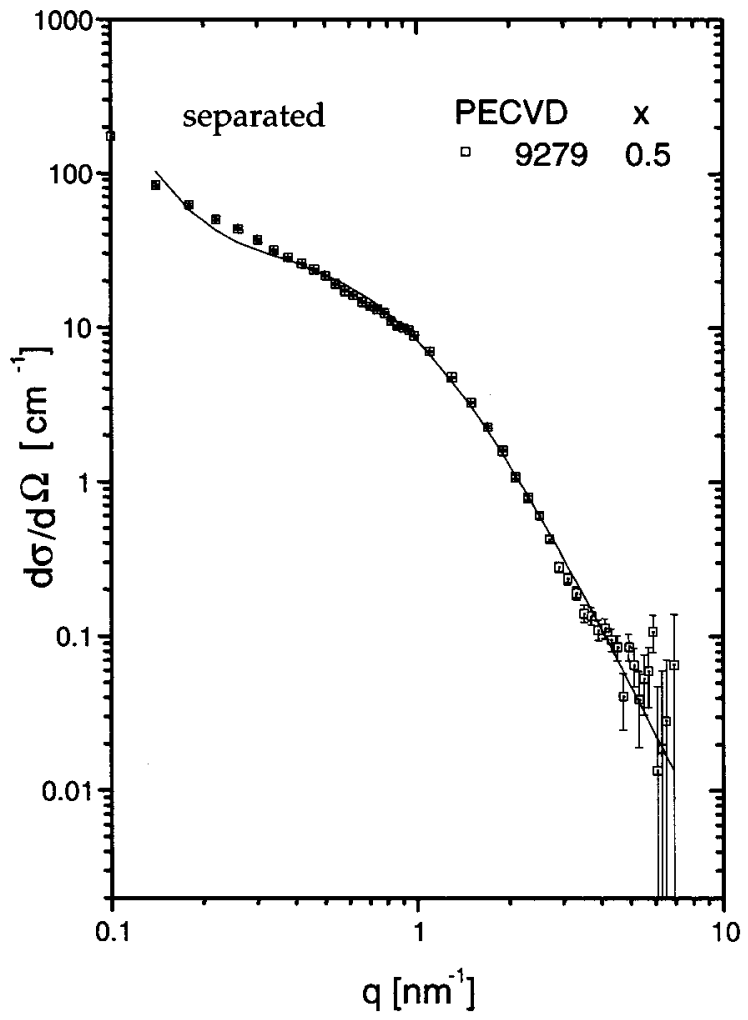

(a)

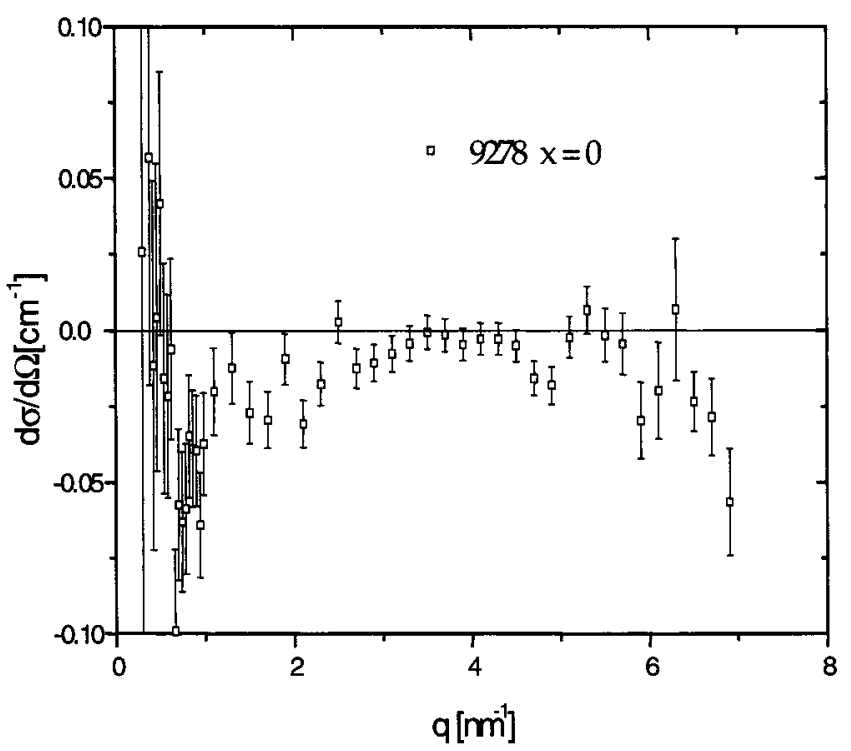

(b)

FIG. 4. Separated scattering intensities from PECVD films with $x=0.50$ (a) and $x=0.0$ (b). Note the conversion to linear scale for the vertical axis of (b).

that the inner surface area between Ge inhomogeneities and the surrounding matrix has reached a maximum value at $x$ $=0.38$. In comparison, the separated scattering curves show a continuous increase with $x$. The shape of the separated scattering curve can be fitted by the Debye correlation model for the Ge concentrations $0.06,0.13,0.23$, and 0.38. Significant differences to the correlation model at $q$ values between 1 and $4 \mathrm{~nm}^{-1}$ occur for $x=0.79$, indicating that the model no longer holds well for this high concentration. The behavior 
TABLE II. Fit results of the six alloys based on Eq. (1). The fitted scattering curves of the alloys with $x$ $=0.06,0.13,0.23,0.38,0.79$, and 0.5 are plotted in Figs. 3 and 4(a).

\begin{tabular}{lcccc}
\hline \hline Sample & $x$ & $a(\mathrm{~nm})$ & $b\left(\mathrm{~cm}^{-1} / \mathrm{nm}^{3}\right)$ & $c\left(\mathrm{~cm}^{-1} / \mathrm{nm}^{4}\right)$ \\
\hline HWCVD-102 & 0.06 & $0.83(4)$ & $1.9(1)$ & 0.0005 \\
HWCVD-100 & 0.13 & $1.00(2)$ & $3.8(1)$ & 0.0074 \\
HWCVD-73 & 0.23 & $0.89(1)$ & $11.1(1)$ & 0.0064 \\
HWCVD-74 & 0.38 & $0.927(4)$ & $33.1(3)$ & 0.0 \\
HWCVD-104 & 0.79 & $1.138(3)$ & $60.3(3)$ & 0.0 \\
PECVD-9279 & 0.50 & $1.018(3)$ & $32.4(2)$ & 0.0269 \\
PECVD-9278 & 0.0 & & & \\
\hline \hline
\end{tabular}

of the hotwire deposited samples may be interpreted as follows: at lower concentrations $(x=0.06$ and 0.13$)$ the $\mathrm{Ge}$ alloying causes inhomogeneities covering length scales from smaller than $1 \mathrm{~nm}$ up to very large structures of $10 \mathrm{~nm}$ and more. At a concentration of 0.23 the larger inhomogeneities are reduced and now Ge inhomogeneities with correlation lengths of about $1 \mathrm{~nm}$ become dominant. This tendency holds on up to concentrations of 0.38 . At higher Ge concentrations, for instance at $x=0.79$, the inner surface area between the Ge-rich phase and the surrounding matrix reaches a maximum and the correlation model gives only a rough approximation of the nanostructure.

Comparison of the total and the separated scattering of the two deposition techniques, HWCVD versus PECVD, shows significant differences in the amount of scattering and of the shape. Both total and separated scattering curves show nearly the same $q$ dependence and the same value of the scattering cross sections at $q$ values larger than $0.5 \mathrm{~nm}^{-1}$ for Ge concentrations of 0.38 (HWCVD) and 0.50 (PECVD). Thus for the sample examined here, the HWCVD technique causes a significantly larger amount of inhomogeneities on a length scale smaller than $20 \mathrm{~nm}$ for the same Ge concentration. At smaller $q$ values or on the length scale larger than 20 $\mathrm{nm}$, the situation is reversed. Finally we note that the total scattering curve of PECVD $a-\mathrm{Si}: \mathrm{H}(x=0)$ shows no energy dependence (separated scattering scatters around zero intensity) with a total scattering $q$ dependence (Fig. 2) which is mainly governed by a $q^{-3}$ behavior, with slight deviations at low and high $q$. The total scattering signal is between 1 and 2 orders smaller with respect to the Ge alloyed PECVD material.

\section{CONCLUSIONS}

ASAXS measurements obtained from $a-\mathrm{Si}_{1-x} \mathrm{Ge}_{x}: \mathrm{H}$ alloys with $x$ between 0.06 and 0.79 reveal clear evidence of nonuniformly distributed $\mathrm{Ge}$ with correlation lengths between 0.8 and $1.1 \mathrm{~nm}$. For the hotwire deposited alloys the amount of separated scattering shows a systematic increase with Ge concentration due to increasing $\mathrm{Ge}$ inhomogeneities. At smaller concentrations $(x=0.06$ and 0.13$)$ a strong increase of the total scattering over the entire $q$ range was observed, indicating an increase of inhomogeneities of different types covering a wide range on the length scale between 1 and $>20 \mathrm{~nm}$. At higher concentrations $(x=0.23)$ the amount of larger inhomogeneities is strongly reduced and replaced by smaller Ge inhomogeneities with correlation lengths of about $1 \mathrm{~nm}$.

From the comparison of HWCVD and PECVD material it was found for similar $x$ that the hotwire deposition technique causes a larger amount of $\mathrm{Ge}$ inhomogeneity on a length scale smaller than $20 \mathrm{~nm}$ and the opposite for larger inhomogeneities.

\section{ACKNOWLEDGMENT}

This work was supported in part by the United States National Renewable Energy Laboratory under Subcontract No. XAK-8-17619-31 to the Colorado School of Mines.

${ }^{1}$ J. Yang, A. Banerjee, and S. Guha, Appl. Phys. Lett. 70, 2975 (1997).

${ }^{2}$ W. Paul, J. H. Chen, E. Z. Liu, A. E. Wetzel, and P. Wickboldt, J. NonCryst. Solids 164-166, 1 (1993).

${ }^{3}$ W. Paul, R. A. Street, and S. Wagner, J. Electron. Mater. 22, 39 (1993).

${ }^{4}$ S. J. Jones, Y. Chen, D. L. Williamson, R. Zedlitz, and G. Bauer, Appl. Phys. Lett. 62, 3267 (1993).

${ }^{5}$ D. L. Williamson, Mater. Res. Soc. Symp. Proc. 377, 251 (1995).

${ }^{6}$ A. R. Middya, S. Ray, S. J. Jones, and D. L. Williamson, J. Appl. Phys. 78, 4966 (1995)

${ }^{7}$ P. Wickboldt, D. Pang, W. Paul, J. H. Chen, F. Zhong, C-C. Chen, J. D. Cohen, and D. L. Williamson, J. Appl. Phys. 81, 6252 (1997).

${ }^{8}$ G. Goerigk and D. L. Williamson, Solid State Commun. 108, 419 (1998).

${ }^{9}$ G. Goerigk and D. L. Williamson, J. Non-Cryst. Solids 281, 181 (2001).

${ }^{10}$ H. Matsumura, J. Appl. Phys. 65, 4396 (1989).

${ }^{11}$ E. C. Molenbroek, A. H. Mahan, and A. Gallagher, J. Appl. Phys. 82, 1909 (1997).

${ }^{12}$ A. H. Mahan and M. Vanecek, in Amorphous Silicon Materials and Solar Cells, AIP Conf. Proc. No. 234, edited by B. L. Stafford (AIP, Woodbury, NY, 1991), p. 195.

${ }^{13}$ A. H. Mahan, R. C. Reedy, E. Iwaniczko, Q. Wang, B. P. Nelson, Y. Xu, A. C. Gallagher, H. M. Branz, R. S. Crandall, J. Yang, and S. Guha, Mater. Res. Soc. Symp. Proc. 507, 119 (1999).

${ }^{14}$ B. P. Nelson, Y. Xu, D. L. Williamson, B. von Roedern, A. Mason, S. Heck, A. H. Mahan, S. E. Schmitt, A. C. Gallagher, J. Webb, and R. Reedy, Mater. Res. Soc. Symp. Proc. 507, 447 (1999).

${ }^{15}$ D. L. Williamson, Y. Xu, and B. P. Nelson, in NCPV Photovoltaics Program Review, AIP Conf. Proc. No. 462, edited by M. Al-Jassim, J. P. Thornton, and J. M. Gee (AIP, Woodbury, NY, 1999), p. 272.

${ }^{16}$ H.-G. Haubold, K. Gruenhagen, M. Wagener, H. Jungbluth, H. Heer, A. Pfeil, H. Rongen, G. Brandenburg, R. Moeller, J. Matzerath, P. Hiller, and H. Halling, Rev. Sci. Instrum. 60, 1943 (1989).

${ }^{17}$ D. T. Cromer and D. Liberman, J. Chem. Phys. 53, 1891 (1970).

${ }^{18}$ D. T. Cromer and D. Liberman, Acta Crystallogr., Sect. A: Cryst. Phys., Diffr., Theor. Gen. Crystallogr. A37, 267 (1981).

${ }^{19}$ P. Debye, H. R. Anderson, and H. Brumberger, J. Appl. Phys. 28, 679 (1957).

${ }^{20}$ G. Porod, in Small Angle X-ray Scattering, edited by O. Glatter and O. Kratky (Academic, New York, 1982), pp. 17-51. 